\title{
O PAPEL DA SEMÂNTICA NO BUSINESS INTELLIGENCE 2.0: Um Exemplo no Contexto de um Programa de Pós-Graduação
}

\author{
Julliano Trindade Pintas, Sean W. M. Siqueira \\ Departamento de Informática Aplicada - Universidade Federal do Estado do Rio de \\ Janeiro (UNIRIO) - Rio de Janeiro, RJ - Brasil \\ \{julliano.pintas, sean\}@uniriotec.br
}

\begin{abstract}
One of the biggest problems in BI nowadays is the latency between the occurrence of an event and the decision made. New concepts that justify the use of the term BI 2.0 promise to reduce such latency. In this work, a semantic system for decision support is presented. It is based on OWL ontologies, SWRL rules and SQWRL query language, showing how the use of Web Semantic technologies can make BI systems more adherent to the business, this way supporting faster and more relevant decisions to the Organization.
\end{abstract}

Resumo. Um dos maiores problemas encontrados no Business Intelligence atual é a latência entre o acontecimento de um evento e a tomada de decisão. Novos conceitos que justificam a utilização do novo termo BI 2.0 prometem reduzir esta latência. Neste trabalho é apresentado um sistema semântico de suporte à decisão baseado em ontologias OWL, regras SWRL e na linguagem de consultas SQWRL, demonstrando como a aplicação de tecnologias da Web Semântica pode tornar os sistemas de BI mais aderentes ao negócio, apoiando, desta forma, decisões mais rápidas e relevantes para a Organização.

\section{Introdução}

Em um mercado cada vez mais globalizado e competitivo, não importando o tipo de negócio que se gerencie, a utilização estratégica da informação é a melhor maneira de se obter vantagens competitivas. Ao contrário, se a informação não é utilizada da maneira correta, compromete-se a própria eficácia da empresa, bem como sua agilidade e eficiência, tornando-a menos competitiva e fadada ao fracasso.

Um dos principais conceitos disponíveis atualmente no que diz respeito à gestão empresarial é o Business Intelligence (BI) ou Inteligência Empresarial. Segundo Bevilacqua e Bitu (2003), BI, tem como principal objetivo disponibilizar informações da maneira e formato correto e no tempo certo para que a empresa possa tomar decisões melhores e mais rápidas. Trata-se de um conjunto de ferramentas e aplicativos que possibilitam aos tomadores de decisão organizar, analisar, distribuir e agir sobre as informações relevantes ao negócio da empresa.

A motivação deste trabalho surgiu da identificação de que as soluções de BI não 
proveem a "inteligência" referida no termo que cunha essas aplicações aos tomadores de decisão, pois se limitam a oferecer funcionalidades genéricas para exploração das montanhas de dados reunidas nos repositórios das organizações. Esse grande volume de dados é submetido ao tomador de decisão, que dispõe unicamente do seu conhecimento pessoal para apoiá-lo durante o processamento analítico, conhecimento este que muitas vezes se mostra insuficiente para fazer os julgamentos corretos em um processamento tão complexo. A seleção, transformação e análise da informação para formar conhecimento ainda dependem exclusivamente das habilidades cognitivas, críticas e teóricas dos tomadores de decisão.

O advento da Web semântica despertou o interesse da comunidade científica para áreas tradicionais de pesquisa, tais como a de Engenharia de Conhecimento, em busca de novas abordagens para tratamento de problemas tradicionais como a integração e a recuperação de informação [Berners-Lee et al. 2001]. O principal objetivo da Web semântica é o de tornar possível a descrição dos recursos disponibilizados na Web ou reunidos em repositórios pessoais de maneira a permitir que agentes localizem conteúdo e realizem inferências sobre esse conteúdo [Berners-Lee et al. 2001]. Para tanto, ontologias vêm sendo utilizadas para agregar descrição semântica e lógica ao conteúdo da Web, de forma a impulsionar a Web corrente para a Web semântica [Fensel 2001].

As organizações modernas, ante os desafios e as oportunidades da sociedade do conhecimento, almejam soluções que permitam a utilização da terminologia e das regras do seu negócio de forma efetiva no processamento analítico [White 2005]. Nesse contexto, uma das maiores deficiências das soluções atuais de BI é a impossibilidade de utilização da semântica do negócio para apoiar a localização, a seleção e a transformação de informação para formar e divulgar conhecimento aos seus colaboradores e parceiros estratégicos. Atualmente, várias empresas já possuem um repositório de conhecimento constituído e agora buscam maneiras de extraírem todo o potencial desses repositórios em busca de diferencial competitivo.

O objetivo deste trabalho é avaliar como a aplicação de tecnologias da Web Semântica pode tornar os sistemas de BI mais aderentes ao negócio, desta forma possibilitando a utilização da semântica do negócio nas atividades de apoio à decisão. Com o sistema de BI mais próximo dos conceitos e regras do negócio é possível pensar em um sistema de BI orientado ao evento e em tempo real.

O contexto de BI 2.0 é apresentado na Seção 2. O estudo de caso no contexto de um Programa de Pós-graduação (PPG) e o sistema desenvolvido para demonstrar a aplicabilidade de tecnologias da Web Semântica são apresentados na Seção 3. O estudo de caso considera a representação de conhecimento através de uma ontologia e a aplicação de regras semânticas sobre esta ontologia. Foi aplicada uma linguagem de consulta semântica para obter, a partir da ontologia, resultados que permitam o suporte à decisão. Este estudo de caso se utiliza de uma aplicação desenvolvida para criar e executar consultas e regras semânticas. Na Seção 4 há uma avaliação do estudo de caso a partir de uma massa de dados de teste, onde são levantadas as vantagens da utilização de tecnologias da Web Semântica em ambientes de BI. Finalmente, na Seção 5 são apresentadas algumas considerações finais. 


\section{BUSINESS INTELLIGENCE 2.0}

Um dos maiores problemas encontrados no BI atual é a latência entre o acontecimento de um evento e a tomada de decisão. Muitas vezes, nos sistemas de suporte à decisão, a informação é disponibilizada muito tarde para ser realmente útil. Quanto mais tarde uma decisão for tomada em relação a um determinado evento, menos útil ela será para a Organização.

Podemos dividir a latência entre o acontecimento de um evento e a tomada de decisão em três partes: latência de dados, latência de análise e a latência de decisão. Segundo Nicholls (2006), o objetivo do BI 2.0 é reduzir todas as três latências, desta forma maximizando o valor de cada decisão tomada. Este aumento de valor representa menor tempo para a identificação de um risco ou uma oportunidade para empresa.

Hoje em dia, existe uma separação grande entre os sistemas analíticos e os sistemas operacionais. Ao buscar a redução da latência de dados, o BI 2.0 busca que os sistemas de BI se tornem mais próximos dos sistemas operacionais criando um sistema analítico em tempo real e orientando a eventos.

A latência de análise pode ser reduzida automatizando a interpretação dos dados, para que as pessoas não precisem olhar para cada item, apenas os problemas. Para isso, é preciso ser capaz de compreender quais eventos empresariais são problemas ou poderão tornar-se futuros problemas [Nicholls 2006].

A latência de decisão pode ser eliminada tanto nas decisões operacionais e táticas automatizando as decisões. O sistema deve interpretar dados em tempo real, dinamicamente.

Este trabalho enfoca a redução da latência de análise através de uma representação formal do conhecimento com o uso de ontologia e lógica, bem como a automação da interpretação dos dados a partir de lógica e regras.

\section{Estudo de Caso}

Para aplicar a solução proposta no estudo de caso foi desenvolvido um sistema baseado na representação de conhecimento através de uma ontologia e definição de regras e consultas semânticas sobre esta ontologia. Além de uma aplicação que permite a criação, edição e execução de regras e consultas semânticas. Este estudo de caso tem como domínio o contexto de um Programa de Pós-graduação (PPG) e tem como objetivo, neste trabalho, exemplificar a utilização de tecnologias semânticas para apoiar as decisões.

O estudo de caso se concentra na redução do tempo de análise. A arquitetura do sistema proposto é ilustrada na Figura 1. Foi utilizada a linguagem OWL para representação da ontologia, SWRL para representação de regras, SQWRL para efetuar consultas semânticas e JAVA para desenvolver a aplicação de criação e execução de consultas.

O sistema desenvolvido deverá apoiar semanticamente análises, através da criação de regras e consultas, quanto à produção pelos componentes do PPG. O mesmo sistema deverá também considerar a produção de outros programas com objetivo de 
comparação, identificação de nível de parcerias entre programas, identificação de oportunidades e nichos não explorados.

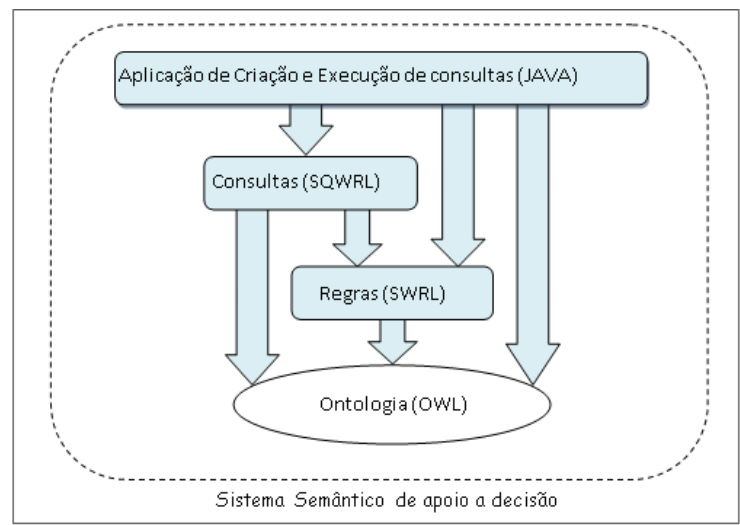

Figura 1. Arquitetura do sistema proposto

Antes da criação da ontologia foi criado um mapa conceitual (Figura 2) para representar de forma visual os conceitos e relações do domínio. Para esta tarefa foi utilizada a suíte CmapTools ${ }^{1}$, que permite aos usuários construir mapas de conceitos que representam o seu entendimento de um domínio de conhecimento. Este ambiente também encoraja a colaboração e compartilhamento durante a construção e manipulação de modelos do conhecimento através de mapas de conceitos (Cmaps).

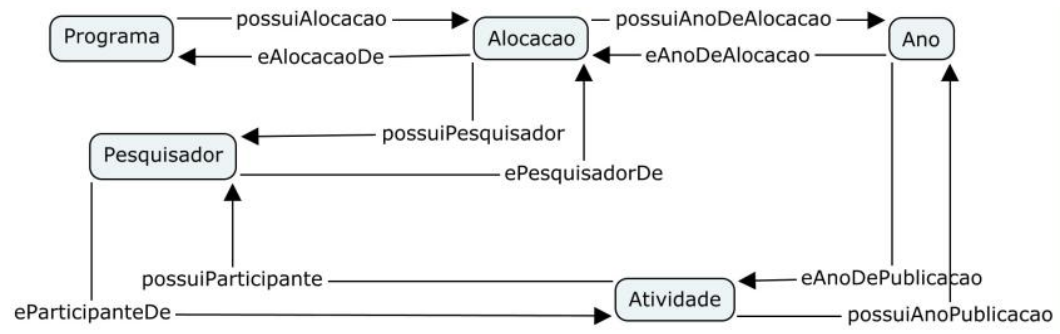

Figura 2. Mapa conceitual da Ontologia

Um PPG pode possuir várias alocações ao longo dos anos. O conceito alocação, neste modelo, representa a alocação de um professor, em um programa, em determinado ano. Um pesquisador, por sua vez, pode realizar várias atividades, cada qual com seu ano de publicação. $O$ conceito atividade representa qualquer trabalho acadêmico que seja passível de publicação. O mapa conceitual representa uma etapa anterior à criação da ontologia por facilitar a visualização dos conceitos a serem representados formalmente na ontologia.

A ferramenta utilizada para modelar a ontologia em OWL foi a Protégé ${ }^{2}$ com o plugin para OWL. Protégé é uma plataforma aberta para a modelagem de ontologias e aquisição de conhecimento. O Plugin OWL pode ser usado para editar ontologias OWL, para acessar raciocinadores de lógica descritiva (DL) e para criar instâncias de classes da ontologia. Como uma extensão do Protégé, o Plugin OWL obtém benefícios de uma

\footnotetext{
${ }^{1}$ http://cmap.ihmc.us/conceptmap.html

${ }^{2} \mathrm{http}: / /$ protege.stanford.edu/
} 
grande comunidade de usuários, uma biblioteca de componentes reutilizáveis e uma arquitetura flexível. O Plugin OWL, portanto, tem potencial para se tornar uma infraestrutura padrão para ontologias na construção de aplicações baseadas em Web Semântica [Knublauch et al. 2004].

Após definidas as classes e relacionamentos não hierárquicos, a ontologia foi implementada em OWL, incluindo os relacionamentos hierárquicos. Para melhor visualização, a hierarquia foi dividida em duas partes. A primeira parte (Figura 3a) mostra a hierarquia no nível mais alto, já a segunda (Figura 3b) mostra apenas a hierarquia composta pelos descendentes da classe atividade.

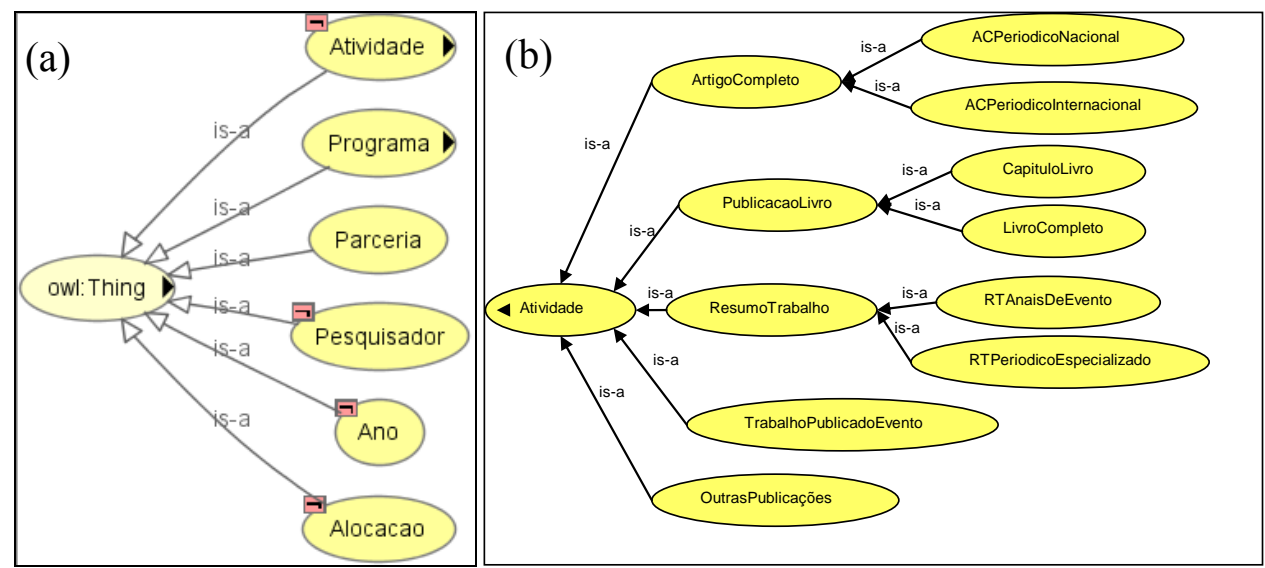

Figura 3. (a) Taxonomia de alto nível da Ontologia; (b) Taxonomia referente a classe Atividade

Uma consulta pode utilizar uma ou mais regras e uma regra pode ser utilizada por diversas consultas. Também é possível a utilização de regras para definir novas regras. A criação de regras pode ajudar bastante na criação de consultas, pois as regras funcionam como sub-consultas, facilitando, desta forma, a definição de consultas mais complexas e o compartilhamento de conhecimento entre consultas distintas.

Essa é uma das principais vantagens da utilização do sistema semântico de suporte à decisão: é possível utilizar o conhecimento explicitado através de regras sobre a ontologia na construção de consultas. Uma vez que uma regra é criada por um usuário especialista do domínio, ela pode ser utilizada em consultas criadas por qualquer outro usuário.

Tanto as regras quanto as consultas são criadas e compartilhadas pelos usuários através da aplicação desenvolvida que será apresentada ao final desta seção. $O$ compartilhamento e reutilização do conhecimento do negócio, representado pelas consultas e regras semânticas, resulta na redução da latência de análise. Outro fator que também resulta na redução desta latência é a maior aderência ao negócio conseguida através da aplicação direta de tecnologias semânticas.

A regra mais utilizada para consultas no estudo de caso é a RegraeProgramaParticipanteDe (Código 1). Esta define como saber que um programa participou de uma atividade. 
Programa(?programa) $\wedge$

possuiAlocacao(?programa, ?alocacao) $\wedge$ possuiAnoDeAlocacao(?alocacao, ?ano) $\wedge$

possuiPesquisador(?alocacao, ?pesquisador) $\wedge$ eParticipanteDe(?pesquisador, ?atividade) $\wedge$

possuiAnoPublicacao(?atividade, ?ano) $\rightarrow$ eProgramaParticipanteDe(?programa, ?atividade)

\section{Código 1. Regra em SWRL - Regra-eProgramaParticipanteDe}

Esta regra define que o programa é considerado participante de uma atividade quando este possui uma alocação com um pesquisador no mesmo ano da publicação da atividade pelo mesmo pesquisador. Depois de definida, esta regra pode ser utilizada por qualquer consulta ou regra que necessite saber sobre a participação de cada programa em cada atividade. Para uma consulta ou regra utilizar esta regra, é só utilizar normalmente a propriedade definida pela regra (eProgramaParticipanteDe) como se fosse qualquer outra propriedade definida diretamente na ontologia.

Todas as consultas foram escritas em SQWRL, sendo utilizadas ou não regras na sua composição. É importante frisar que por ainda estar em desenvolvimento, a linguagem SQWRL não possibilita a realização de consultas com maior nível de complexidade. Algumas evoluções já foram propostas no primeiro artigo publicado sobre a linguagem pelos seus criadores O'Connor e Das (2009), como, por exemplo, a utilização de conjuntos para obter resultados equivalentes ao que se obteria em um sistema mundo fechado como em um banco de dados relacional tradicional. Porém, essas propostas ainda não foram, até o momento da publicação deste trabalho, implementadas pelo principal motor de regras $\mathrm{Jess}^{3}$.

A Consulta-ProducaoPessoalAno (Código 2) é uma das consultas do estudo de caso, exibindo a Produção total em quantidade de publicações por ano para cada pesquisador. Caso um pesquisador não possua nenhuma publicação em um determinado ano, não haverá no resultado desta consulta uma linha referente a aquele pesquisador e ano. A última cláusula, que define os nomes das colunas, se mostra importante, pois na aplicação desenvolvida é exibido o nome de cada coluna de resultado dinamicamente de acordo com o nome definido na consulta.

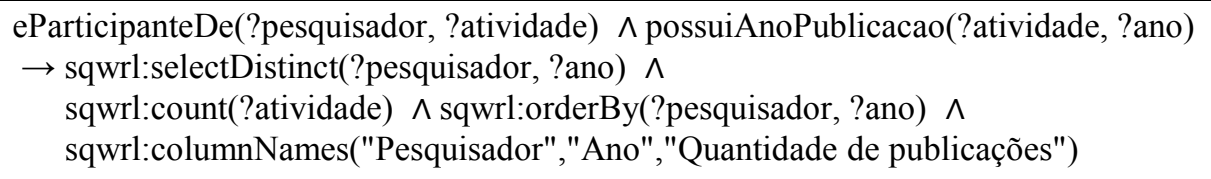

Código 2. Consulta em SQWRL - Consulta-ProducaoPessoalAno

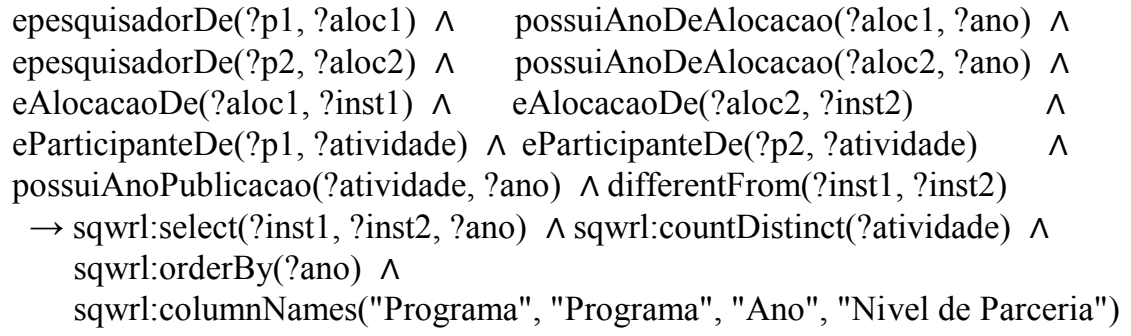

Código 3. Consulta em SQWRL - Consulta-NivelParceriaPorAno

\footnotetext{
${ }^{3}$ http://www.jessrules.com/
} 
Outra consulta definida é a Consulta-NivelParceriaPorAno (Código 3), que mostra o nível de parceria entre cada par de instituições a cada Ano. Esta consulta é muito importante, pois possibilita ver a evolução ao longo dos anos da cooperação entre instituições distintas na produção bibliográfica. Isto permite a busca de novas parcerias e manutenção de outras.

Foi criada outra consulta semelhante a essa última, chamada ConsultaNivelParceria, que mostra o nível de parceria entre os programas no total, ou seja, sem desacoplar por ano.

As regras e consultas definidas não são exaustivas, representam exemplos de regras e consultas que podem ser criadas para esta ontologia. Outras consultas e regras podem ser criadas. De modo a permitir a criação, edição, execução e compartilhamento de consultas e regras, foi desenvolvida uma aplicação em Java para Web.

O desenvolvimento desta aplicação foi de grande valia para avaliar, na próxima seção, a viabilidade de construir aplicações de suporte à decisão com a arquitetura semelhante à proposta neste trabalho.

O framework Java Server Faces foi escolhido para este desenvolvimento. A aplicação foi dividida em um esquema de abas. A primeira aba (Figura 4) lista todas as regras e consultas já criadas e permite a edição ou execução de cada uma delas. Para melhor visualização, as consultas e regras são paginadas em grupos de cinco, mas é permitida a alteração do modo de visualização para exibir todas as consultas sem paginação. Esta aba também permite a criação de novas regras ou consultas.

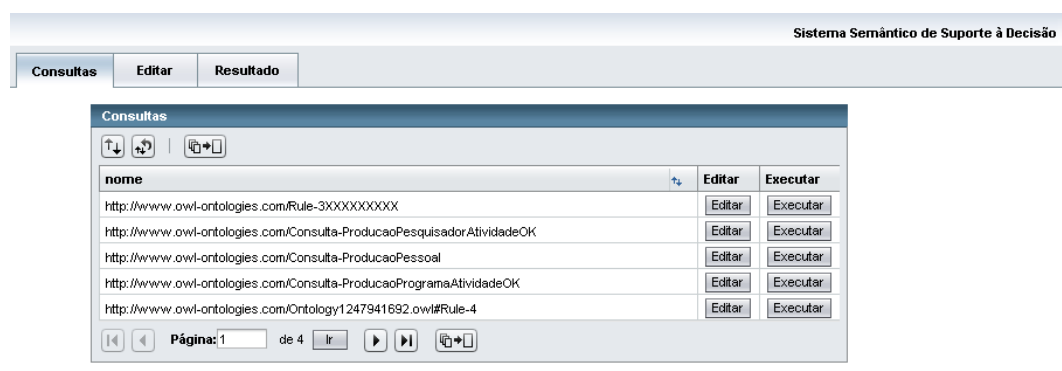

Figura 4. Aba de listagem de regras e consultas

A segunda aba (Figura 5) permite a edição e criação tanto de regras quanto de consultas. Os mesmos controles da tela são utilizados pra regra e consultas, o que vai definir se vai ser uma regra ou uma consulta são os built-ins utilizados na construção. Para facilitar a criação de regras e consultas, é fornecida uma árvore exibindo a hierarquia da ontologia e permitindo a inserção de classes na regra/consulta. Também é fornecida uma listagem com todos os built-ins SWRL e SQWRL.

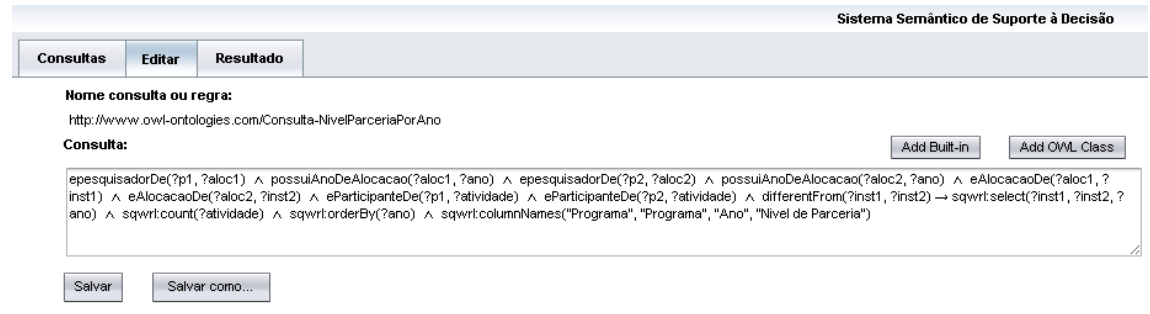

Figura 5. Aba de edição de consultas e regras 
A terceira aba (Figura 6) é exclusiva para exibição dos resultados de execução de consultas. A visualização dos resultados pode ser feita de dois modos: modo tabela e modo gráfico.

A tabela que exibe os resultados é totalmente dinâmica. Foi implementado um mecanismo que ajusta automaticamente a quantidade, títulos e largura das colunas de resultado de acordo com o resultado da consulta executada. Por isso é importante a definição dos nomes das colunas nas consultas.

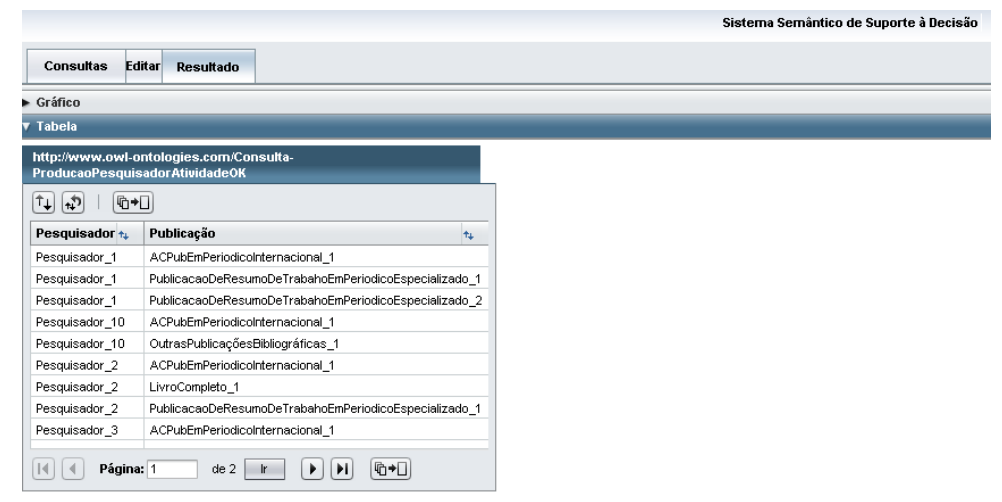

Figura 6. Aba resultado no modo de visualização em tabela

O mecanismo de ordenação múltipla é acionado ao clicar no título de uma coluna. Ao clicar no título de uma coluna já ordenada, é feita alteração no modo de ordenação (ascendente/descendente).

No modo de visualização de resultado em gráfico, o gráfico pode ser configurado para atender melhor cada consulta. É possível escolher o tipo de gráfico desejado e quais colunas que vão ser utilizadas na construção do gráfico. Os tipos de gráficos disponíveis são barra, pizza e linha.

O sistema exibe a caixa que solicita os parâmetros para configuração do gráfico que nesta aplicação permite apenas uma série de valores. São solicitados o tipo de gráfico desejado, o campo que vai ser utilizado como rótulo e o campo que vai ser utilizado como valor, que deve ser um campo numérico. Por padrão, o gráfico é configurado como sendo do tipo pizza, o primeiro campo como sendo o rótulo e o segundo como sendo o valor.

$\mathrm{Na}$ implementação da aplicação foram utilizadas as APIs disponibilizadas pelo Protégé e seus plugins para acessar e manipular a ontologia, principalmente a SWRLAPI, que é um ambiente de desenvolvimento para trabalhar com regras SWRL [O'Connor et al. 2008]. Para definir e executar regras e consultas, foi utilizado o motor de regras $\mathrm{JESS}^{4}$. Para criar os gráficos de resultado em tempo de execução, foi utilizado o Google Chart $\mathrm{API}^{5}$.

\footnotetext{
${ }^{4}$ http://www.jessrules.com/

${ }^{5} \mathrm{http} / / /$ code.google.com/intl/pt-BR/apis/chart/image_charts.html
} 


\section{Avaliação}

Para avaliar o funcionamento do sistema semântico de suporte à decisão, foi utilizada uma massa de dados de testes. A escolha de utilizar dados de teste ao invés de dados reais deve-se ao fato que os dados de teste puderam ser projetados para cobrir todos os casos envolvidos nas regras e consultas criadas. Os dados são representados como instâncias da ontologia e suas respectivas propriedades. O processo de ETL, nesse caso, faria a carga de dados em forma de instâncias da ontologia. Logo, para utilizar dados reais, seria necessário implementar um processo ETL de alguma fonte específica, como, por exemplo, do Lattes, para a ontologia.

Os dados de teste consistem em 4 anos, 3 programas, 10 pesquisadores, 16 atividades e 30 alocações. Foram criadas consultas em SQWRL específicas para apresentar os dados da massa de teste.

A consulta TodosPesquisadores retornou todas as instâncias da classe pesquisador. $\mathrm{O}$ código 4 mostra esta consulta.

$$
\text { Pesquisador(?x) } \rightarrow \text { sqwrl:select(?x) }
$$

\section{Código 4. Consulta em SQWRL - Consulta-TodosPesquisadores}

A consulta TodosAnos retornou todas as instâncias da classe Ano. A consulta TodasAtividades retornou todas as instâncias das sub-classes da classe atividade e seus respectivos anos de publicação. Algumas consultas utilizam o ano de publicação em sua construção, logo para que seja possível comparar os resultados destas consultas com os dados de entrada, foi importante exibir o ano de publicação de cada atividade. $\mathrm{O}$ código 5 mostra o código desta consulta.

\begin{tabular}{|lccccc}
\hline $\begin{array}{l}\text { Atividade(?atividade) } \\
\text { sqwrl:select(?atividade, ?ano) }\end{array} \wedge$ & possuiAnoPublicacao(?atividade, & ?ano) & $\rightarrow$ \\
\hline
\end{tabular}

\section{Código 5. Consulta em SQWRL - Consulta-TodasAtividades}

No caso das alocações, o que é necessário saber são o ano, o pesquisador e o programa relacionados a cada alocação. A consulta TodasAlocações retorna exatamente isto, os nomes das instâncias que são propriedades de cada alocação criada.

A consulta TodasParticipantes retorna o cruzamento de cada atividade com seus participantes. A consulta ProducaoPesquisadorAtividade exibe o cruzamento dos pesquisadores com suas atividades realizadas, sem diferenciar a instituição ou ano em que ocorreu publicação da atividade.

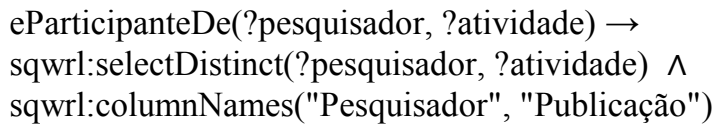

\section{Código 6. Consulta em SQWRL - Consulta-ProducaoPesquisadorAtividade}

A consulta ProducaoPessoal exibe a quantidade de publicações realizadas agrupando por pesquisador, também sem diferenciar a instituição ou ano em que ocorreu publicação. A consulta ProducaoPessoalAno exibe a quantidade de publicações 
realizadas agrupando por pesquisador e ano, ou seja, exibe quantas publicações foram realizadas por cada pesquisador em cada ano.

A consulta ProducaoProgramaAtividade exibe o cruzamento dos programas com suas publicações, sem diferenciar o ano em que ocorreu publicação. Esta consulta possui, apesar da semelhança, maior complexidade do que a consulta ProducaoPesquisadorAtividade, pois a ontologia não representa diretamente a participação dos programas nas atividades, apenas dos pesquisadores. Para isto, foi criada a regra Regra-eProgramaParticipanteDe.

O conhecimento definido na regra pode ser reutilizado em várias consultas, e até na definição de outras regras. Neste caso, uma vez que alguém definiu através da regra como saber se um programa participou de uma atividade, qualquer usuário que necessite criar uma consulta que envolva este conceito poderá utilizar esta regra na construção de sua consulta.

A consulta ProducaoPrograma é semelhante à consulta ProducaoPessoal, só que exibe a quantidade de publicações realizadas agrupando por programa, também sem diferenciar ano em que ocorreu a publicação.

A consulta NivelParceriaPorAtividade exibe o nível de parceria, ou seja, a quantidade de programas envolvidos na realização de cada atividade. Deste modo, é possível definir quais trabalhos tiveram maior ou menor nível de cooperação entre programas durante sua realização.

A consulta NivelParceriaPorAno mostra o nível de parceria entre cada par de programas ao longo dos anos. Com isto é possível analisar a evolução da parceria do programa em questão com outros programas ao longo dos anos.

Com esta consulta é possível responder várias perguntas, como por exemplo: Qual o programa de pós-graduação que mais cooperou no último ano com o PPG que está sendo analisado? Existe algum programa que vem cooperando, cada ano que passa, menos com o programa em análise? De acordo com nível de parceria dos anos anteriores é possível fazer uma projeção para o próximo ano, através de algum mecanismo de projeção, como média móvel ou regressão linear. Desta forma é possível responder qual programa possui a melhor ou pior projeção de cooperação com a instituição em análise para o próximo ano. Para responder essas e outras perguntas, é interessante utilizar o mecanismo de ordenação múltipla de colunas da tabela e o gráfico configurável que estão presentes na aplicação desenvolvida.

A consulta NivelParceria (Código 7) mostra o nível de parceria total entre cada par de programas. Com isto é possível analisar quais programas que possuíram maior ou menor número de trabalhos desenvolvidos em conjunto.

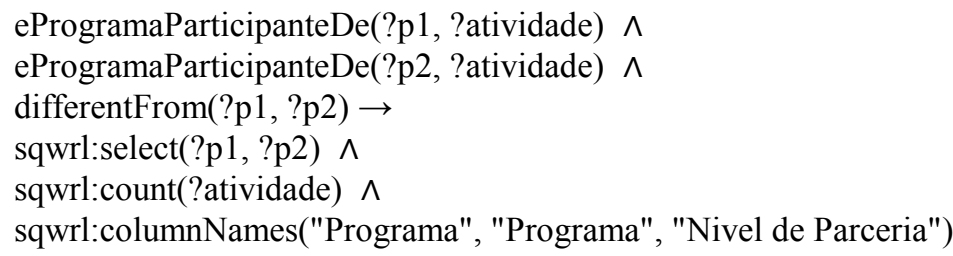

Código 7. Consulta em SQWRL - Consulta-NivelParceria 
As consultas NivelParceriaPorAno e NivelParceria são bidirecionais, logo apresentam duplicações em seus resultados. Não foi encontrado nenhum método para evitar, utilizando SQWRL, estas duplicações. O que foi feito é o uso da cláusula differentFrom para evitar o cálculo do nível de parceria de um programa com ele próprio. Para esta cláusula funcionar como desejado, foi necessário definir que todas as instâncias da classe programa representam indivíduos diferentes no mundo real através da tag owl:AllDifferent.

\section{Conclusão}

Os esforços para criação e avaliação do exemplo foram grandes, uma vez que a linguagem SQWRL é recente e está em pleno desenvolvimento, possuindo poucos estudos de casos e referências de uso em outras aplicações. Apesar de ainda não permitir consultas muito complexas, notou-se que esta recente linguagem oferece um potencial muito grande com consultas no formato da lógica de predicados que referenciam diretamente as entidades e regras da ontologia.

Houve também um esforço para que a aplicação desenvolvida ficasse independente da ontologia utilizada nesse exemplo, ou seja, a aplicação de gerenciamento e execução de regras e consultas poderá ser evoluída e utilizada para qualquer outro repositório de conhecimento baseado OWL. Vale também ressaltar que a aplicação possui uma arquitetura totalmente baseada na Web, onde foi utilizado o framework Java Server Faces.

Através da avaliação dos resultados das consultas e do desenvolvimento da aplicação de criação e execução de consultas, observa-se que consultas e regras semânticas podem oferecer resultados equivalentes a consultas realizadas em um SGBD, tornando possível implementar (utilizando consultas semânticas) vários mecanismos de suporte à decisão, como dashboards, relatórios e até ferramentas equivalentes às ferramentas OLAP tradicionais, em que seja possível realizar operações OLAP, como Drill down/up e slice and dice. Um estudo mais aprofundado destes aspectos poderia validar esta hipótese.

Também, através da avaliação do estudo de caso, é possível notar que, apesar dessa equivalência de resultados de consultas, a utilização de tecnologias da Web semântica apresenta a vantagem de permitir a utilização da terminologia e das regras do seu negócio de forma efetiva no processamento analítico. Com a utilização da semântica, diversas ferramentas de BI poderão reutilizar e compartilhar regras e conceitos do negócio, assim diminuindo a latência de análise e criando um ambiente de BI mais aderente ao negócio.

Para tomar decisões com maior segurança e rapidez, é essencial que o usuário do sistema de BI entenda os conceitos e regras por trás da aplicação. Nesta análise, nota-se que em um sistema semântico de suporte à decisão os conceitos e regras, além de apoiar na criação e manutenção de consultas, podem servir como metadados para os tomadores de decisão.

Logo, na análise do exemplo desenvolvido, conclui-se que a utilização de tecnologias da Web Semântica em sistemas de BI é viável e tem grande potencial para 
facilitar, através da manutenção de regras e consultas semânticas, o processo de tomada de decisão.

Os possíveis trabalhos futuros se dividem em dois principais grupos. O primeiro está relacionado ao desenvolvimento de uma ferramenta que apóie este processo de ETL específico, em que os dados não são carregados para um Data Warehouse tradicional, mas para uma ontologia.

O segundo grupo de trabalhos futuros envolve a evolução do sistema semântico de suporte à decisão desenvolvido, com a adição de novas funcionalidades que favoreçam a tomada de decisão. São possibilidades de evolução deste sistema o desenvolvimento de:

- Uma funcionalidade que permita a construção de consultas de forma visual.

- Uma funcionalidade que permita a definição semântica de scorecards.

- Um esquema de dashboards e relatórios baseados em consultas semânticas.

- Uma funcionalidade que permita através de consultas semânticas a sugestão ou automatização da tomada de decisão.

- Funcionalidades que permitam operações equivalentes às presentes em ferramentas OLAP tradicionais, como Drill down/up e slice and dice.

\section{Referências}

Berners-Lee, T., Hendler, J. e Lassila, O. (2001) The Semantic Web. In Scientific American, maio de 2001.

Bevilacqua, J. F. e Bitu, Y. A. (2003) Business Intelligence (BI) e a abordagem de Gestão Balanced Scorecard (BSC) na Organização. Monografia de MBA em Gestão de Sistemas de Informação. Universidade Católica de Brasília. 54f.

Fensel, D. (2001), Ontologies: silver bullet for knowledge management and electronic commerce. Berlin: Springer-Verlag, 2001.

Knublauch, H., Fergerson, R. W., Noy, N. F. e Musen, M. A. (2004) The Protégé OWL Plugin: An Open Development Environment for Semantic Web Applications. In The Semantic Web - ISWC 2004, Vol. 3298 (2004), pag. 229-243.

Nicholls, C. (2006), In Search of Insight, SeeWhy Software Limited.

O'Connor, M. J., Nyulas, C. I., Shankar, R. D., Das, A. K. e Musen, M. A. (2008) The SWRLAPI: A Development Environment for Working with SWRL Rules. In OWL: Experiences and Directions (OWLED), Fifth International Workshop. Karlsruhe, Germany.

O'Connor, M. J., DAS, A. K. (2009) SQWRL: a Query Language for OWL. In OWL: Experiences and Directions (OWLED), Fifth International Workshop. Chantilly, VA.

White, C. The Role of Business Intelligence in Knowledge Management. B Eye. Mar. 2005. Disponível em: <http://www.b-eye-network.com/view/720>. Acesso em: 20 de Fevereiro de 2011. 